\title{
Numerical Solution of Reynolds Equations
}

\author{
for Forest Fire Spread
}

\author{
Valeri Perminov \\ Belovo Branch of Kemerovo State University, Sovetskay, 41 \\ 652600, Belovo, Kemerovo region, Russia \\ pva@belovo. kemsu.ru
}

\begin{abstract}
In the present paper it is planned to develop mathematical model for description of heat and mass transfer processes at crown forest fire spread. The paper suggested in the context of the general mathematical model of forest fires [1] gives a new mathematical setting and method of numerical solution of a problem of a forest fire spread. It was based on numerical solution of two dimensional Reynolds equations for the description of turbulent flow taking into account for diffusion equations chemical components and equations of energy conservation for gaseous and condensed phases. To obtain discrete analogies a method of controlled volume [2] was used. Numerical solution of this problem during surface and crown fires in exemplified heat energy release in the forest fire front was found.
\end{abstract}

\section{Introduction}

The research was made by means of the mathematical modeling methods of physical processes [3]. It was based on numerical solution of two dimensional Reynolds equations for the description of turbulent flow taking into account for diffusion equations chemical components and equations of energy conservation for gaseous and condensed phases. Powerful up-currents of gas occur in surface and crown forest fires, causing entrapment of heated gaseous combustion products of forest fuels in the ground layer. In this context, a study - mathematical modeling - of the conditions of forest fire spreading that would make it possible to obtain a detailed picture of the change in the velocity, temperature and component concentration fields with time, and determine as well as the limiting conditions of forest fire propagation is of interest.

\section{Forest Fire Equations}

Let us examine a plane problem of radiation-convection heat and mass exchange of forest fuels in all forest strata with gaseous combustion products and radiation from the tongue of flame of the surface forest fire. The surface fire source is modeled as a plane layer of burning forest fuels with known temperature and increasing area of burning. It is assumed that the forest during a forest fire can be modeled as a two-temperature multiphase non-deformable porous reactive medium [1]. Let there be a so-called "ventilated" forest massif, in which the volume of fractions of condensed forest fuel phases, consisting of dry organic matter, water in liquid state, solid pyrolysis products, and ash, can be neglected compared to the volume fraction of gas phase (components of air and gaseous pyrolysis products). To describe the transfer of energy by 
radiation we use a diffusion approximation, while to describe convective transfer controlled by the wind and gravity, we use Reynolds equations.

Let the coordinate reference point $x_{1}, x_{2} x_{3}=0$ be situated at the center of the forest fire source, axis $0 x_{3}$ directed upward, axis $0 x_{1}$ and $0 x_{2}$ directed parallel to the Earth's surface $\left(\mathrm{x}_{1}\right.$ - to the right in the direction of the unperturbed wind speed, $\mathrm{x}_{2}-$ perpendiculary of the wind direction).

Because of the horizontal sizes of forest massif more than height of forest $-\mathrm{h}$, system of equations of general mathematical model of forest fire [1] was integrated between the limits from height of the roughness level - 0 to h. Besides, suppose that

$$
\int_{0}^{h} \phi d x_{3}=\bar{\phi} h
$$

$\dddot{\phi}$ - average value of $\phi$. The problem formulated above is reduced to a solution of the following system of equations:

$$
\begin{aligned}
& \frac{\partial \rho}{\partial t}+\frac{\partial}{\partial x_{j}}\left(\rho v_{j}\right)=Q-\left(\dot{m}^{-}-\dot{m}^{+}\right) / h, j=1,2, i=1,2 \\
& \rho \frac{d v_{i}}{d t}=-\frac{\partial p}{\partial x_{j}}+\frac{\partial}{\partial x_{j}}\left(-\rho{\overrightarrow{v_{i}^{\prime}}}_{j}^{\prime}\right)-\rho s c_{d} v_{i}|\vec{v}|-\rho g_{i}-Q v_{i}+ \\
& +\left(\tau^{-}-\tau^{+}\right) / h \text {; } \\
& \rho c_{p} \frac{d T}{d t}=\frac{\partial}{\partial x_{j}}\left(-\rho c_{p} \overline{\left.v_{j}^{\prime} T^{\prime}\right)}+q_{5} R_{5}-\alpha_{v}\left(T-T_{s}\right)+\left(q_{T}^{-}-q_{T}^{+}\right) / h ;(3)\right. \\
& \rho \frac{d c_{\alpha}}{d t}=\frac{\partial}{\partial x_{j}}\left(-\rho \overline{v_{j}^{\prime} c_{\alpha}^{\prime}}\right)+R_{5 \alpha}-Q c_{\alpha}+\left(J_{\alpha}^{-}-J_{\alpha}^{+}\right) / h, \alpha=1,3 \\
& \frac{\partial}{\partial x_{j}}\left(\frac{c}{3 k} \frac{\partial U_{R}}{\partial x_{j}}\right)-k\left(c U_{R}-4 \sigma T_{S}^{4}\right)+\left(q_{R}^{-}-q_{R}^{+}\right) / h=0 \\
& \sum_{i=1}^{4} \rho_{i} c_{p i} \varphi_{i} \frac{\partial T_{S}}{\partial t}=q_{3} R_{3}-q_{2} R_{2}+k\left(c U_{R}-4 \sigma T_{S}^{4}\right)+\alpha_{V}\left(T-T_{S}\right) \\
& \rho_{1} \frac{\partial \varphi_{1}}{\partial t}=-R_{1}, \rho_{2} \frac{\partial \varphi_{2}}{\partial t}=-R_{2} \\
& \rho_{3} \frac{\partial \varphi_{3}}{\partial t}=\alpha_{C} R_{1}-\frac{M_{C}}{M_{1}} R_{3}, \rho_{4} \frac{\partial \varphi_{4}}{\partial t}=0 \\
& \sum_{\alpha=1}^{3} c_{\alpha}=1, p_{e}=\rho R T \sum_{\alpha=1}^{3} \frac{c_{\alpha}}{M_{\alpha}}, \vec{v}=\left(v_{1}, v_{2}\right), \vec{g}=(0,0, g)
\end{aligned}
$$




$$
\begin{aligned}
& Q=\left(1-\alpha_{c}\right) R_{1}+R_{2}+\frac{M_{c}}{M_{1}} R_{3}, R_{51}=-R_{3}-\frac{M_{1}}{2 M_{2}} R_{5}, \\
& R_{52}=v\left(1-\alpha_{c}\right) R_{1}-R_{5}, R_{53}=0 .
\end{aligned}
$$

The system of equations (1) - (9) must be solved taking into account the following initial and boundary conditions:

$$
\begin{aligned}
& t=0: v_{1}=0, v_{2}=0, T=T_{e}, c_{\alpha}=c_{\alpha e} ; \\
& x_{1}=x_{10}: v_{1}=V_{e}, v_{2}=0, T=T_{e}, c_{\alpha}=c_{\alpha e} ; \\
& x_{1}=x_{1 e}: \frac{\partial v_{1}}{\partial x_{1}}=0, \frac{\partial v_{2}}{\partial x_{1}}=0, \frac{\partial c_{\alpha}}{\partial x_{1}}=0, \frac{\partial T}{\partial x_{1}}=0 ; \\
& x_{2}=x_{20}: \frac{\partial v_{1}}{\partial x_{2}}=0, \frac{\partial v_{2}}{\partial x_{2}}=0, \frac{\partial c_{\alpha}}{\partial x_{2}}=0, \frac{\partial T}{\partial x_{2}}=0 ; \\
& x_{2}=x_{2 e}: \frac{\partial v_{1}}{\partial x_{2}}=0, \frac{\partial v_{2}}{\partial x_{2}}=0, \frac{\partial c_{\alpha}}{\partial x_{2}}=0, \frac{\partial T}{\partial x_{2}}=0 ;
\end{aligned}
$$

It should be noted that the condition of symmetry is used in this statement instead of (13)

$$
x_{2}=x_{20}: \frac{\partial v_{1}}{\partial x_{2}}=0, v_{2}=0, \frac{\partial c_{\alpha}}{\partial x_{2}}=0, \frac{\partial U_{R}}{\partial x_{2}}=0, \frac{\partial T}{\partial x_{2}}=0,
$$

Because of the patterns of flow and distribution of all scalar functions are symmetrical relative to the axis $0 \mathrm{x}_{2}$.

Here and above $\frac{\mathrm{d}}{\mathrm{d} t}$ is the symbol of the total (substantial) derivative; $\alpha_{\mathrm{v}}$ is the coefficient of heat and mass exchange; $\mathrm{t}$ is time; $x_{i}, v_{i},(\mathrm{i}=1,2)$ are the Cartesian coordinates ( $\mathrm{x}$ and $\left.\mathrm{y}\right)$ and the velocity components; index $\alpha=1,2,3$, where 1 corresponds to the density of oxygen, 2 to carbon monoxide $\mathrm{CO}, 3$ - concentration of inert components of air; $V_{e}$ - equilibrium wind velocity.

The thermodynamic, thermophysical and structural characteristics correspond to the forest fuels in the canopy of a pine forest [1]. To define source terms, which characterize inflow (outflow of mass) in a volume unit of the gas-dispersed phase, the following formulae (9) were used for the rate of forming of the gas-dispersed mixture $\mathrm{Q}$, outflow of oxygen $R_{51}$ and changing carbon monoxide $R_{52}$. The source of ignition is defined as a function of time and turned off after the forest fire initiation.

Thus, the solution of the system of equations (1) - (9) with initial and boundary conditions (10) - (15) may result in defining the fields of velocity, temperature, component concentrations and radiation density. 
The system of equations (1)-(8) contains terms associated with turbulent diffusion, thermal conduction, and convection, and needs to be closed. The components of the tensor of turbulent stresses, $\rho v_{i}^{\prime} v_{j}^{\prime}$ as well as the turbulent fluxes of heat and mass $\overline{\rho c_{p} v_{i}^{\prime} T^{\prime}}, \overline{\rho v_{i}^{\prime} c_{\alpha}^{\prime}}$ are written in terms of the gradients of the average flow properties using the formulas:

$$
\begin{aligned}
& -\rho v_{i}^{\prime} v_{j}^{\prime}=\mu_{t}\left(\frac{\partial v_{i}}{\partial x_{j}}+\frac{\partial v_{j}}{\partial x_{i}}\right)-\frac{2}{3} K \delta_{i j}, \\
& -\rho c_{p} v_{j}^{\prime} T^{\prime}=\lambda_{t} \frac{\partial T}{\partial x_{j}},-\rho v_{i}^{\prime} c_{\alpha}^{\prime}=\rho D_{t} \frac{\partial c_{\alpha}}{\partial x_{i}}, \\
& \lambda_{t}=\mu_{t} c_{p} / \operatorname{Pr}_{T}, \rho D_{t}=\mu_{t} / S c_{T}, \mu_{t}=c_{\mu} \rho K^{2} / \varepsilon,
\end{aligned}
$$

where $\mu_{\mathrm{t}}, \lambda_{\mathrm{t}}, D_{\mathrm{t}}$ are the coefficients of turbulent viscosity, thermal conductivity, and diffusion, respectively; $\mathrm{Pr}_{\mathrm{t}}, \mathrm{Sc}_{\mathrm{t}}$ are the turbulent Prandtl and Schmidt numbers, which were assumed to be equal to 1 . The coefficient of dynamic turbulent viscosity is determined using the localequilibrium model of turbulence [1].

\section{The Method of Solution}

The boundary-value problem (1) - (15) was solved numerically using the method of splitting according to physical processes. In the first stage, the hydrodynamic pattern of flow and distribution of scalar functions was calculated. The system of ordinary differential equations of chemical kinetics obtained as a result of splitting was then integrated. A discrete analog for equations (1) - (9) was obtained by means of the control volume method using the SIMPLE algorithm $[2,3]$.

The accuracy of the program was checked by the method of inserted analytical solutions. Analytical expressions for the unknown functions were substituted in (1)-(9) and the closure of the equations were calculated. This was then treated as the source in each equation. Next, with the aid of the algorithm described above, the values of the functions used were inferred with an accuracy of not less than $1 \%$. The effect of the dimensions of the control volumes on the solution was studied by diminishing them. The time step for chemical stage was selected automatically.

\section{Typical Calculated Results and Discussion}

Fields of velocity, temperature, component mass fractions, volume fractions of phases were obtained numerically. The distribution of basic functions shows that the process goes through the some stages. As a result of heating of forest fuel elements, moisture evaporates, and pyrolysis occurs accompanied by the release of gaseous products, which then ignite. and burn away in the forest canopy.

In the vicinity of the source of heat and mass release, heated air masses and products of pyrolysis and combustion float up. At $V_{e} \neq 0$, the wind field in the forest canopy interacts with the gas-jet obstacle that forms from the surface forest fire source and from the ignited forest 
canopy base. On the windward side the movement of the air flowing past the ignition region accelerates (Fig. 1).

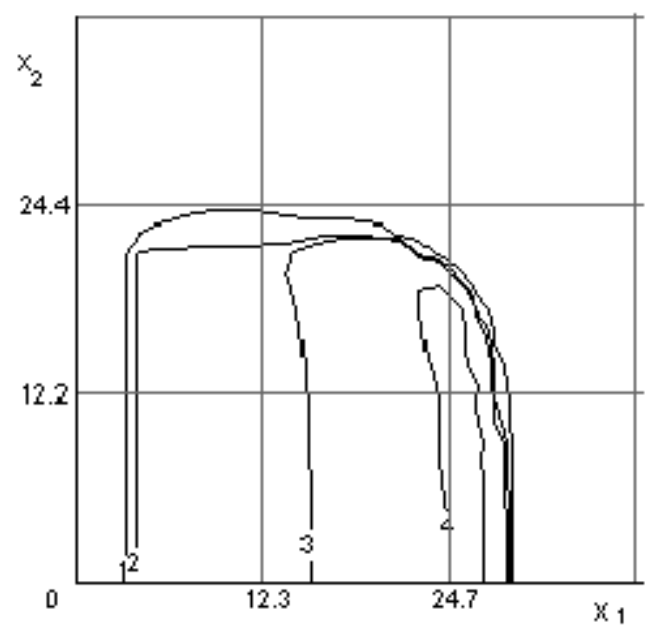

Fig. 1. Isotherms of the forest fire for $\mathrm{t}=5 \mathrm{~s}$ and $V_{e}=5 \mathrm{~m} / \mathrm{s}$ : $1-\bar{T}=1.5,2-\bar{T}=2.6 ., 3-\bar{T}=3.5,4-T=5$.

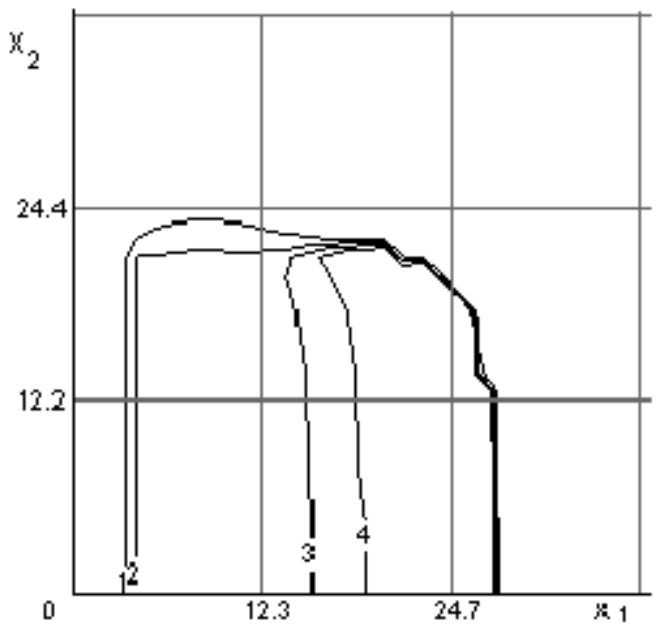

Fig. 2. . Isotherms of the solid phase for $\mathrm{t}=5 \mathrm{~s}$ and $V_{e}=5 \mathrm{~m} / \mathrm{s}$ : $1-\bar{T}_{s}=2.6,2-\bar{T}_{s}=3.5,3-T_{s}=5$. 


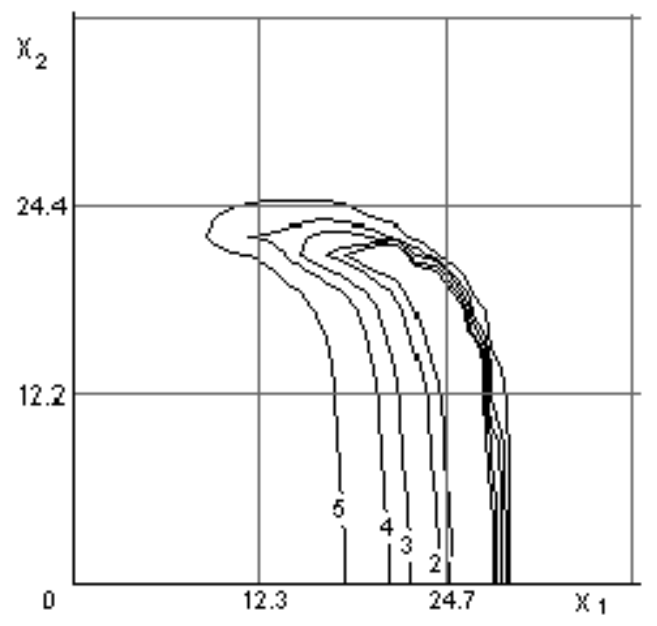

Fig.3. The distribution of oxygen $\bar{c}_{1}$ at $\mathrm{t}=5 \mathrm{~s}$ and $V_{e}=5 \mathrm{~m} / \mathrm{s}$ : $1-\bar{c}_{1}=0.5,2-\bar{c}_{1}=0.6,3-\bar{c}_{1}=0.75,4-\bar{c}_{1}=0.85,5-\bar{c}_{1}=0.9$.

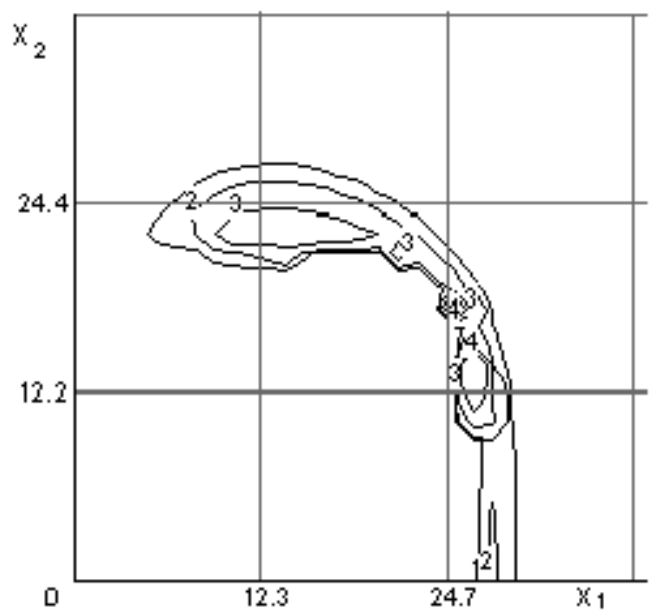

Fig. 4. The distribution of $\bar{c}_{2}$ at $\mathrm{t}=5 \mathrm{~s}$ and $V_{e}=5 \mathrm{~m} / \mathrm{s}$ : $1-\bar{c}_{2}=0.01,2-\bar{c}_{2}=0.03,3-\bar{c}_{2}=0.12,4-\bar{c}_{2}=0.4$. 


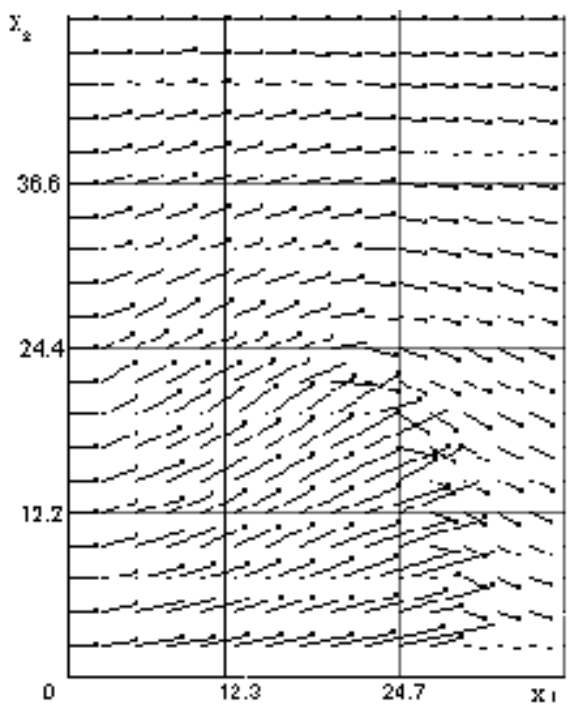

Fig. 5.

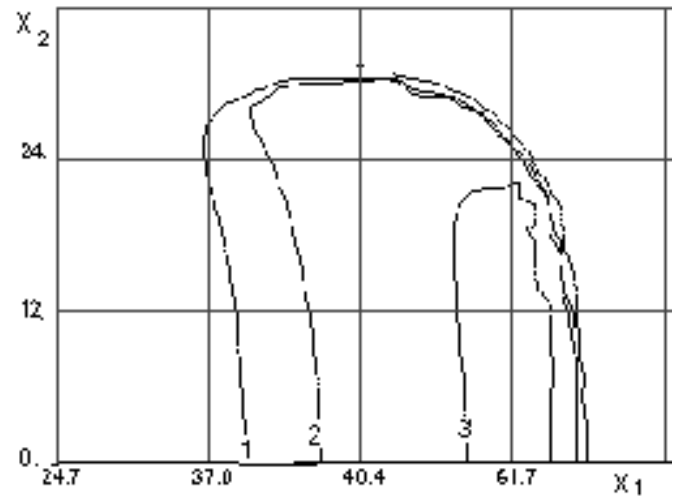

Fig. 6. Isotherms of the forest fire for $\mathrm{t}=10 \mathrm{~s}$ and $V_{e}=5 \mathrm{~m} / \mathrm{s}$ : $1-\bar{T}=2.6,2-\bar{T}=3.5 ., 3-\bar{T}=5$. 


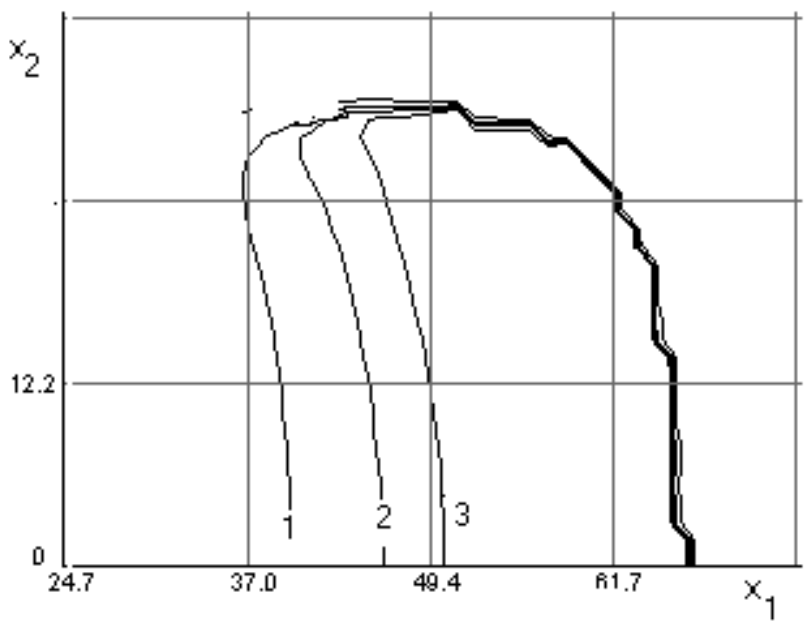

Fig. 7. Isotherms of the solid phase for $\mathrm{t}=10 \mathrm{~s}$ and $V_{e}=5 \mathrm{~m} / \mathrm{s}$ : $1-\bar{T}_{s}=2.6,2-\bar{T}_{s}=3.5,3-\bar{T}_{s}=5$.

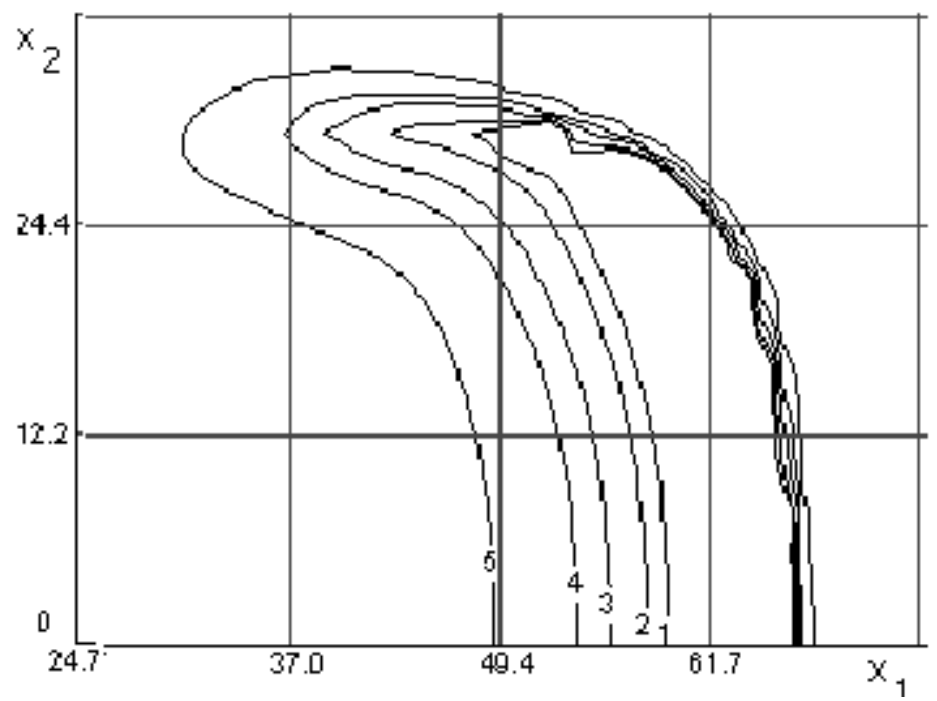

Fig. 8. The distribution of oxygen $\bar{c}_{1}$ at $\mathrm{t}=10 \mathrm{~s}$ and $V_{e}=5 \mathrm{~m} / \mathrm{s}$ : $1-\bar{c}_{1}=0.5,2-\bar{c}_{1}=0.6,3-\bar{c}_{1}=0.75,4-\bar{c}_{1}=0.85,5-\bar{c}_{1}=0.9$. 


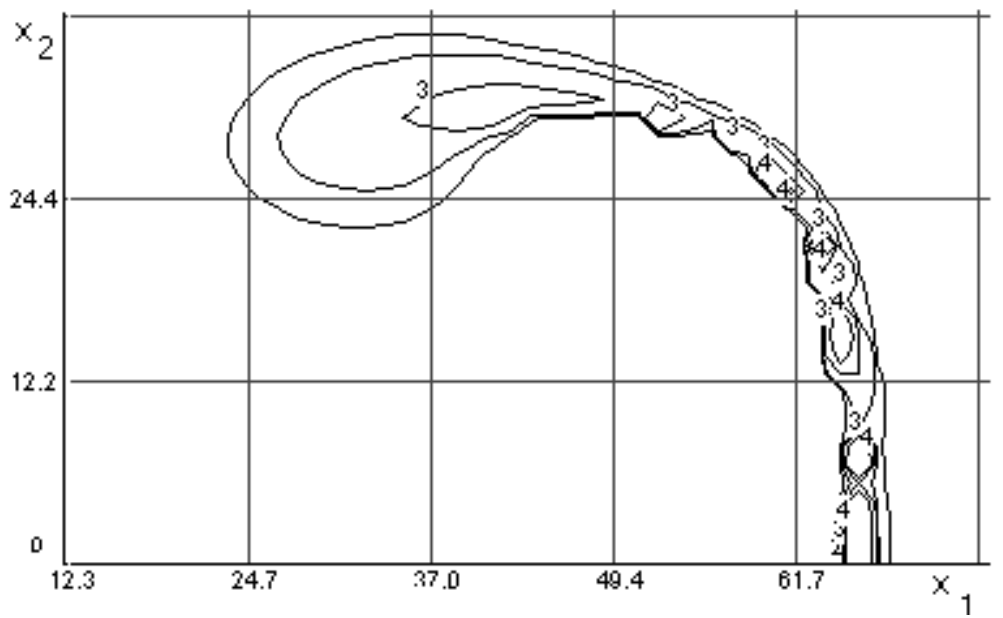

Fig. 9. The distribution of $\bar{c}_{2}$ at $\mathrm{t}=10 \mathrm{~s}$ and $V_{e}=5 \mathrm{~m} / \mathrm{s}$ : $1-\bar{c}_{2}=0.01,2-\bar{c}_{2}=0.03,3-\bar{c}_{2}=0.12,4-\bar{c}_{2}=0.4$.

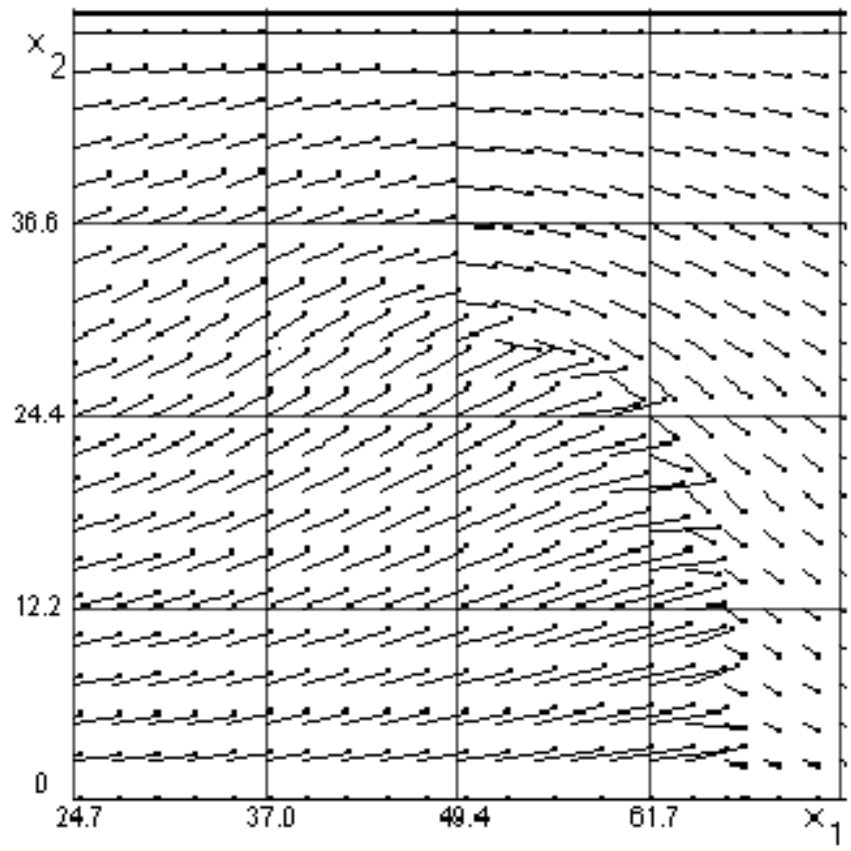

Fig. 10. 
Fig. 2, 3 and 4 present the distribution of temperature $\bar{T}\left(\bar{T}=T / T_{e}, T_{e}=300 \mathrm{~K}\right)$ for gas and condensed phases, oxygen $\bar{c}_{1}$, volatile combustible products of pyrolysis $\bar{c}_{2}$ concentrations $\left(\bar{c}_{\alpha}=c_{\alpha} / c_{1 e}, c_{1 e}=0.23\right.$ ) for wind velocity $V_{e}=5 \mathrm{~m} / \mathrm{s}: t=5 \mathrm{~s}$ after the beginning of forest combustible materials ignition. We can note that the isotherms is moved in the forest canopy and deformed by the action of wind. Similarly, the fields of component concentrations are deformed. The distribution of the same functions (Fig. 6-11) show that the forest fire begins spread. High temperature zone is removed in $x_{1}-$ direction (Fig. 6). Of interest is the investigation of the influence forest combustible materials properties (moisture, density and etc) and wind velocity on the rate of front fire spread.

The obtained results are agree with the laws of physics and experimental data [1].

\section{References}

1. Grishin A.M.: Mathematical modeling forest fire and new methods fighting them, Nauka, Novosibirsk (1992)

2. Patankar S.V.: Numerical heat transfer and fluid flow. Hemisphere Publishing Corporation, New York (1984)

3. Perminov V.A., Mathematical Modeling of Crown and Mass Forest Fires Initiation With the Allowance for the Radiative - Convective Heat and Mass Transfer and Two Temperatures of Medium, Ph.D Thesis, Tomsk State University, Tomsk, (1995) 